\title{
Uniaxial stress and Zeeman spectroscopy of the 3.324-eV Ge-related photoluminescence in ZnO
}

\author{
J. Cullen, ${ }^{1}$ K. Johnston, ${ }^{2,3}$ E. McGlynn, ${ }^{1}$ M. O. Henry, ${ }^{1, *}$ D. Dunker, ${ }^{4}$ D. R. Yakovlev, ${ }^{4,5}$ and M. Bayer ${ }^{4}$ \\ ${ }^{1}$ School of Physical Sciences, National Centre for Plasma Science and Technology, Dublin City University, Glasnevin, Dublin 9, Ireland \\ ${ }^{2}$ PH Department, ISOLDE/CERN, 1211 Geneva 23, Switzerland \\ ${ }^{3}$ Technische Physik, Universität des Saarlandes, Building E2.6, P.O. Box 151150, 66041 Saarbrücken, Germany \\ ${ }^{4}$ Experimentelle Physik 2, Technische Universität Dortmund, 44227 Dortmund, Germany \\ ${ }^{5}$ Ioffe Physical-Technical Institute, Russian Academy of Sciences, 194021 St. Petersburg, Russia
}

(Received 15 February 2013; published 15 April 2013)

\begin{abstract}
Recently observed photoluminescence (PL) in $\mathrm{ZnO}$, positioned at $3.324 \mathrm{eV}$ and known to be related to $\mathrm{Ge}$ impurities, is investigated here by uniaxial stress and Zeeman spectroscopy measurements. The 3.324-eV PL line shifts but does not split under uniaxial stress both parallel and perpendicular to the $c$ axis, indicating trigonal defect symmetry. This reinforces the findings of prior work that the defect center is related to a substitutional Ge impurity in ZnO. Applied magnetic fields result in linear splittings of the line into two components for fields parallel and perpendicular to the $c$ axis. This result, combined with the temperature dependence of the Zeeman spectra, enables the line to be assigned to neutral donor bound-exciton recombination, most likely at a partially compensated $\mathrm{Ge}_{\mathrm{Zn}}$ double-donor impurity.
\end{abstract}

DOI: 10.1103/PhysRevB.87.165202

PACS number(s): 78.55.-m, 71.55.-i, 78.55.Et, 71.35.-y

\section{INTRODUCTION}

The potential uses of the wide band-gap semiconductor $\mathrm{ZnO}$ have been discussed extensively elsewhere. ${ }^{1}$ However, the development of homojunction devices is hampered by persistent difficulties with regard to producing material with well-controlled $p$-type conductivity. In addition to the lack of a reliable, reproducible $p$-type doping process, there are unresolved issues regarding a variety of impurities in $\mathrm{ZnO}$, including the chemical nature of the impurities that give rise to some I lines in the band-edge luminescence. However, a number of recent studies have successfully identified specific I lines with neutral or ionized donor bound-exciton (DBX) recombination at $\mathrm{Al}, \mathrm{Ga}$, and In impurities, ${ }^{2-4}$ with the result that the majority of the I lines first investigated by Reynolds et $a .^{5}$ are now well understood.

In contrast to the clear understanding of the main I line luminescence, there is less clarity regarding new exciton recombination features observed deeper in the photoluminescence (PL) spectrum, which are different in some respects from the I lines. ${ }^{6,7}$ These new features have been labeled Y lines in Ref. 7, while one of the lines in this region was labeled DD by Schildknecht et al., ${ }^{8}$ a notation style that was continued by Johnston et al. ${ }^{3,6}$ Of these $\mathrm{DD} / \mathrm{Y}$ lines, the case of $\mathrm{DD}_{1} / \mathrm{Y}_{0}$ has been most thoroughly investigated, encompassing temperature dependence, lifetimes, uniaxial stress, magneto-optical, and luminescence imaging measurements. ${ }^{7}$ While conclusive identification of the $\mathrm{DD}_{1} / \mathrm{Y}_{0}$ defect core was not made in Ref. 7 , the authors provided strong arguments in favor of a model based on two adjacent donors acting, under illumination, as a single binding center for a neutral DBX. In contrast to $\mathrm{DD}_{1} / \mathrm{Y}_{0}$, for which no information about the chemical constituents of the defect is available, there is a clear chemical identification for the case of $\mathrm{DD}_{2}$. Experimental studies of $\mathrm{ZnO}$ :Ge were made by some of the present authors in the course of the study of $\mathrm{ZnO}$ implanted with radioactive As and $\mathrm{Ga}^{3,6}$ In both cases, the line labeled $\mathrm{DD}_{2}$, which appeared at $3.324 \mathrm{eV}$, was shown to be related to $\mathrm{Ge}$. Although theoretical investigations have been made of the role of the group IV impurities $\mathrm{Si}$ and $\mathrm{Ge}$ in
$\mathrm{ZnO},{ }^{9}$ with both proposed as likely $n$-type dopants occupying substitutional lattice cation sites, a direct assignment of $\mathrm{DD}_{2}$ to neutral DBX recombination at Ge impurities could not be made definitively.

In this paper, we extend our investigations of the $\mathrm{DD}_{2}$ line in $\mathrm{ZnO}$ :Ge with a view to determining the defect symmetry and the nature of the exciton recombination. Uniaxial stress measurements are reported along two different crystal directions (parallel and perpendicular to the $c$ axis) in order to examine any orientational or electronic degeneracy exhibited by the center. Zeeman spectroscopy is performed in order to make an assignment of the feature to (neutral or ionized) DBX or acceptor bound-exciton recombination. The results are compared and contrasted throughout with the corresponding data for $\mathrm{DD}_{1} / \mathrm{Y}_{0}$ and the $\mathrm{I}$ lines.

\section{EXPERIMENTAL DETAILS}

Hydrothermally grown single-crystal samples of $\mathrm{ZnO}$, obtained from Tokyo Denpa Ltd., were doped with Ge using ion implantation at a nominal dose of $1 \times 10^{13}$ ions $/ \mathrm{cm}^{2}$ with an ion beam energy of $100 \mathrm{keV}$. Samples were then annealed in an $\mathrm{O}_{2}$ atmosphere at $750^{\circ} \mathrm{C}$ for $30 \mathrm{~min}$ in order to remove residual implantation damage in the crystal. Three samples with different crystalline orientations were used in the paper. The sample dimensions were either $10 \times 10 \times 0.5$ or $10 \times 4 \times 0.5 \mathrm{~mm}^{3}$.

For the majority of the PL measurements, PL emission was generated using the $325-\mathrm{nm}$ line of a $\mathrm{HeCd}$ laser with an excitation density of $\sim 200 \mathrm{~mW} / \mathrm{cm}^{2}$. For general measurements, the PL was analyzed using a 1-m grating spectrometer (SPEX 1704 with a resolution of $0.01 \mathrm{~nm}$ ) equipped with a photomultiplier tube (Hamamatsu model R3310-02) in photon counting mode and cooled by a Peltier system EMI FACT50 cooler to approximately $-20^{\circ} \mathrm{C}$. An iHR 320 spectrometer with a resolution of $0.07 \mathrm{~nm}$ was used to record spectra of samples under uniaxial stress; the detector was a Peltiercooled Andor Newton electron-multiplying charge-coupled 
device (CCD) cooled to $-30^{\circ} \mathrm{C}$. For PL measurements at the On-Line Isotope Mass Separator facility (ISOLDE) in the European Organisation for Nuclear Research (CERN), the luminescence was analyzed by a SPEX $0.75-\mathrm{m}$ grating spectrometer with a resolution of $0.01 \mathrm{~nm}$ equipped with a liquid nitrogen-cooled Jobin-Yvon CCD detector. In all cases, closed-cycle cryostats (either a SHI-950-5 or a SHI-950, both from Janis Corp.) were used to cool the samples to temperatures in the range $4-20 \mathrm{~K}$.

Magneto-optical measurements of the Zeeman splitting were made using a split-coil magnet cryostat from Oxford Instruments generating magnetic fields up to $7 \mathrm{~T}$; as an excitation source, the second harmonic (using an LBO crystal) of an optically pumped (Verdi $10 \mathrm{~W}$ laser from Coherent) Ti:sapphire laser from Tekhnoscan was used with a wavelength of $355 \mathrm{~nm}$ and $~ 50-\mathrm{mW}$ power, and the PL was analyzed with a Horiba U1000 double monochromator, at a resolution of $0.01 \mathrm{~nm}$, equipped with a R943-02 Hamamatsu photomultiplier tube. Sample temperatures in the range 5-10 K were used in the magneto-optical measurements.

For uniaxial stress measurements, vertically aligned samples were mounted in shallow slots cut to the thickness of the samples in the faces of two stainless steel pistons in a stress apparatus described in Ref. 10. A piezoelectric load cell (Bofors KRA-1) produced an output voltage proportional to the force applied on the sample through a push rod. A steel ball bearing was used at the base of the load cell to transfer the force of the spring-loading mechanism without transferring the twisting motion of the threaded bar to the piston. The sample temperature for the uniaxial stress measurements was $10 \mathrm{~K}$.

\section{RESULTS}

\section{A. Photoluminescence}

All Tokyo-Denpa $\mathrm{ZnO}$ samples used in this paper produced similar PL spectra. The PL spectrum of an as-received $c$-plane sample is shown in Fig. 1(a) alongside that of a $c$-plane sample doped with Ge via ion implantation, as detailed earlier. The $\mathrm{DD}_{2}$ emission feature $\sim 3.324 \mathrm{eV}$ was observed in all three samples implanted with Ge that we produced for this paper and in other $\mathrm{ZnO}: \mathrm{Ge}$ samples produced in earlier studies. ${ }^{3,6}$ Also observed near the $\mathrm{DD}_{2}$ feature and at slightly higher energies is the line labeled $\mathrm{DD}_{1}$ in the figure, which was the subject of extensive study recently. ${ }^{2,7}$ We use the label $\mathrm{DD}_{1} / \mathrm{Y}_{0}$ for this line hereafter. The changes in the near-band-edge DBX emission lines under annealing are not part of this paper, and no further comment is made on these lines except to compare their shifts under stress and their intensities relative to their longitudinal optical (LO) replicas with those of the main subject of the paper, the $\mathrm{DD}_{2}$ line.

The low-temperature PL spectrum in Fig. 1(b) shows $\mathrm{DD}_{2}$ and some associated features at lower energies that are present only in Ge-doped samples. In addition to a prominent LO phonon replica peak $\sim 3.252 \mathrm{eV}$ (at a separation of $\sim 72 \mathrm{meV}$ from $\mathrm{DD}_{2}$ ), there are two features centered $\sim 3.262 \mathrm{eV}$. This coincides with the position at which an LO phonon replica of $\mathrm{DD}_{1} / \mathrm{Y}_{0}$ should occur, but the latter is extremely weak. ${ }^{7}$ The low thermal binding energy exhibited by $\mathrm{DD}_{2}{ }^{6}$ is also observed for these features (shown in the Fig. 1(b) inset), and we
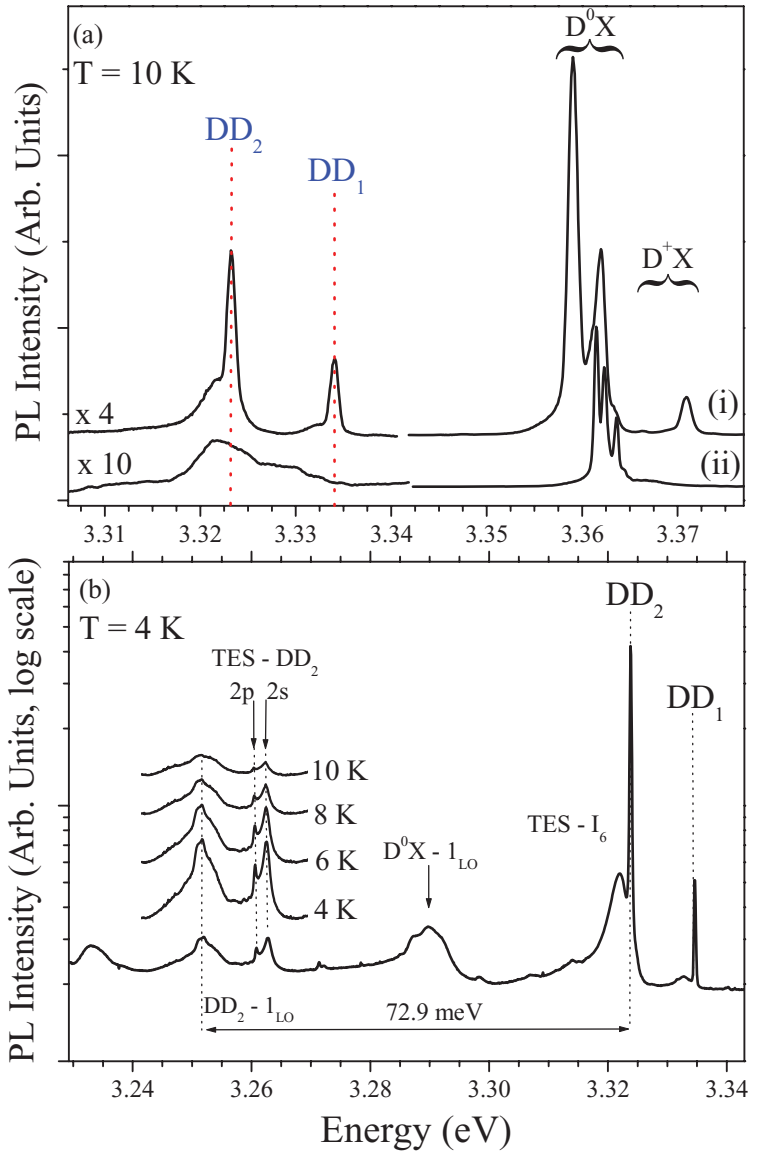

FIG. 1. (Color online) (a) PL spectra of $\mathrm{ZnO}$ (i) after implantation with Ge and (ii) as received. Shown in more detail in panel (b) is the region of the spectrum where the $\mathrm{DD}_{2}$ line is observed in $\mathrm{ZnO}: \mathrm{Ge}$. Spectra of the TES lines and the $1_{\mathrm{LO}}$ replica of $\mathrm{DD}_{2}$ for different temperatures in the range $4-10 \mathrm{~K}$ are included in panel (b).

attribute the lines to two-electron satellite (TES) lines of $\mathrm{DD}_{2}$. Our assignment of PL features to TES transitions involving $\mathrm{DD}_{2}$ is based on donor behavior for the $\mathrm{DD}_{2}$ center; this is corroborated later from Zeeman spectroscopy measurements.

In order to facilitate subsequent discussion, we include in Table I the principal spectroscopic data for $\mathrm{DD}_{2}$, along with the corresponding values, where they are available, for several of the I lines and other DD/Y lines reported in Ref. 7. The data include the line energy, the exciton localization with respect to the free exciton energy 3.3759-eV $E_{\text {loc }}$, the thermal binding energy $E_{a}$, and the TES line separations $\Delta E(1 s-2 p)$ and $\Delta E(2 s-2 p)$. The Huang-Rhys parameter $S$ values for the intensity ratios of the $\mathrm{LO}$ replicas relative to their respective zero-phonon line are also listed, as are the shift rates under stress $\delta E / \delta P$, described later.

\section{B. Uniaxial stress spectroscopy}

Uniaxial stress measurements can provide unique insight into the orientational and electronic degeneracy of exciton recombination centers. ${ }^{11}$ For this paper, uniaxial compression was applied (1) along the $c$-axis direction [0001], for which the overall crystal symmetry is unchanged under stress, and (2) perpendicular to the $c$ axis along the [1-210] direction, which 
TABLE I. Spectroscopic data for the main I lines and Y lines reported in Ref. 7 are given in rows 2 to 8 and columns 2 to 8 . The data for $\mathrm{DD}_{2}$ in the final row and the shift rate for $\mathrm{I}_{6}$ in the final column were obtained in this paper or reported in Ref. 6. Missing values are due either to the measurements not having been reported to date (-) or to the line intensities being too low (•). The $\Delta E(1 s-2 p)$ and $\Delta E(2 s-2 p)$ TES identifications for $\mathrm{DD}_{1} / \mathrm{Y}_{0}$ are given as provisional (Ref. 7).

\begin{tabular}{|c|c|c|c|c|c|c|c|c|}
\hline Line & $\begin{array}{c}\text { Energy } \\
(\mathrm{eV})\end{array}$ & $\begin{array}{c}E_{\mathrm{loc}} \\
(\mathrm{meV})\end{array}$ & $\begin{array}{c}E_{a} \\
(\mathrm{meV})\end{array}$ & $\begin{array}{c}\Delta E(1 s-2 p) \\
(\mathrm{meV})\end{array}$ & $\begin{array}{c}\Delta E(2 s-2 p) \\
(\mathrm{meV})\end{array}$ & $\begin{array}{c}S \\
\left(\mathrm{I}_{\mathrm{LO}} / \mathrm{I}_{\mathrm{ZPL}}\right)\end{array}$ & $\begin{array}{c}\delta E / \delta P \| c \\
(\mathrm{meV} / \mathrm{GPa})\end{array}$ & $\begin{array}{l}\delta E / \delta P \perp c \\
(\mathrm{meV} / \mathrm{GPa})\end{array}$ \\
\hline $\mathrm{I}_{4}$ & 3.3628 & 13.1 & 13 & 34.1 & -0.9 & 0.052 & 2.79 & - \\
\hline $\mathrm{I}_{6}$ & 3.3612 & 15.8 & 15 & 38.8 & 0.8 & 0.058 & 2.84 & -1.81 \\
\hline $\mathrm{I}_{8}$ & 3.3598 & 16.1 & 16 & 42.1 & 1.4 & $\bullet$ & 2.99 & - \\
\hline $\mathrm{I}_{9}$ & 3.3567 & 19.2 & 19 & 50.6 & 4.0 & 0.067 & 3.37 & - \\
\hline$Y_{2}$ & 3.3465 & 29.4 & • & $\bullet$ & $\bullet$ & $\bullet$ & 1.95 & - \\
\hline $\mathrm{Y}_{1}$ & 3.3363 & 39.6 & 12 & $\bullet$ & $\bullet$ & $\bullet$ & 2.15 & - \\
\hline $\mathrm{DD}_{1} / \mathrm{Y}_{0}$ & 3.3328 & 43.1 & 12 & 63.4 & 0.8 & 0.004 & 2.09 & - \\
\hline $\mathrm{DD}_{2}$ & 3.3238 & 53.2 & 2.9 & 62.9 & 1.8 & 0.02 & 2.93 & -1.37 \\
\hline
\end{tabular}

reduces the crystal symmetry from $\mathrm{C}_{6 \mathrm{v}}$ to $\mathrm{C}_{2 \mathrm{v}}$. Samples of different orientations were purchased in order to accommodate different stress directions.

A comprehensive study of the behavior of four I lines and three Y lines for stress parallel to the $c$ axis was carried out by Wagner et al. ${ }^{7}$ In order to make a direct comparison of our $\mathrm{DD}_{2}$ data with that full set of data, we normalized the shift rate of $I_{6}$ in our measurements to that given for $I_{6}$ in Ref. 7 as a calibration for our stress values. With this calibration, a neighboring line $\mathrm{I}_{8 \mathrm{a}}$ is found to shift at $2.88 \mathrm{meV} / \mathrm{GPa}$, close to the value of $2.99 \mathrm{meV} / \mathrm{GPa}$ reported for $\mathrm{I}_{8}$ in Ref. 7, which validates the calibration. Our uniaxial stress data for $\mathrm{DD}_{2}$ are presented in Fig. 2. The slight curvature in the line shifts for low stresses is present in similar studies of $\mathrm{ZnO}{ }^{7,12}$ The spectra of $\mathrm{DD}_{2}$ for stress parallel to the $c$ axis are shown in Fig. 2(a), where a shift toward higher energies is observed, the data for which are plotted in Fig. 2(b). The shift rate of the line for this stress direction is $\delta E / \delta P=+2.93 \mathrm{meV} / \mathrm{GPa}$. There is little change in the line width or intensity for this stress direction.

The spectra for stress perpendicular to the $c$ axis, along the [1-210] direction, are shown in Fig. 2(c), and the data are plotted in Fig. 2(d). A small negative shift is observed for this stress direction compared to the larger positive shift rate for stress parallel to the $c$ axis. Data for the low-stress regime only are available due to the tendency for samples to break for stresses along the [1-210] direction. The shift rate $\delta E / \delta P=-1.35 \mathrm{meV} / \mathrm{GPa}$ is estimated for stresses up to $150 \mathrm{MPa}$. The $\mathrm{I}_{6}$ line (not shown) is found to shift at a rate of $-0.81 \mathrm{meV} / \mathrm{GPa}$. Detailed PL data for individual I lines under stress perpendicular to the $c$ axis have not been published elsewhere to date, although a negative shift for this stress direction was observed for unresolved luminescence lines as part of a photoreflectance study in $1970 .{ }^{12}$

\section{Zeeman spectroscopy}

Magneto-optical measurements were made for two geometries: (1) $\mathbf{B} \| \mathbf{c}$ and $\mathbf{E} \perp \mathbf{c}$ and (2) $\mathbf{B} \perp \mathbf{c}$ and $\mathbf{E} \| \mathbf{c}$. As strong selection rules restrict the number of allowed transitions in each case, a schematic energy level diagram is given in Fig. 3(a), which indicates the allowed and forbidden transitions of a DBX for the two cases; this is used later in the discussion of the data.
Figure 3 also shows the PL spectra of the $\mathrm{DD}_{2}$ line under zero field and for magnetic field values of 3,5 , and $7 \mathrm{~T}$ for the field parallel (b) and perpendicular (c) to the $c$ axis. Although the line lies on top of a varying background luminescence, a clear splitting is observed for $\mathbf{B} \perp \mathbf{c}$. For $\mathbf{B} \| \mathbf{c}$, the magnetic field effect is weaker, with the line only broadening. The two components for $\mathbf{B} \perp \mathbf{c}$ in Fig. 3(c) maintain approximately the same intensity ratio as the field increases (i.e., no thermalization is observed between the two lines as their

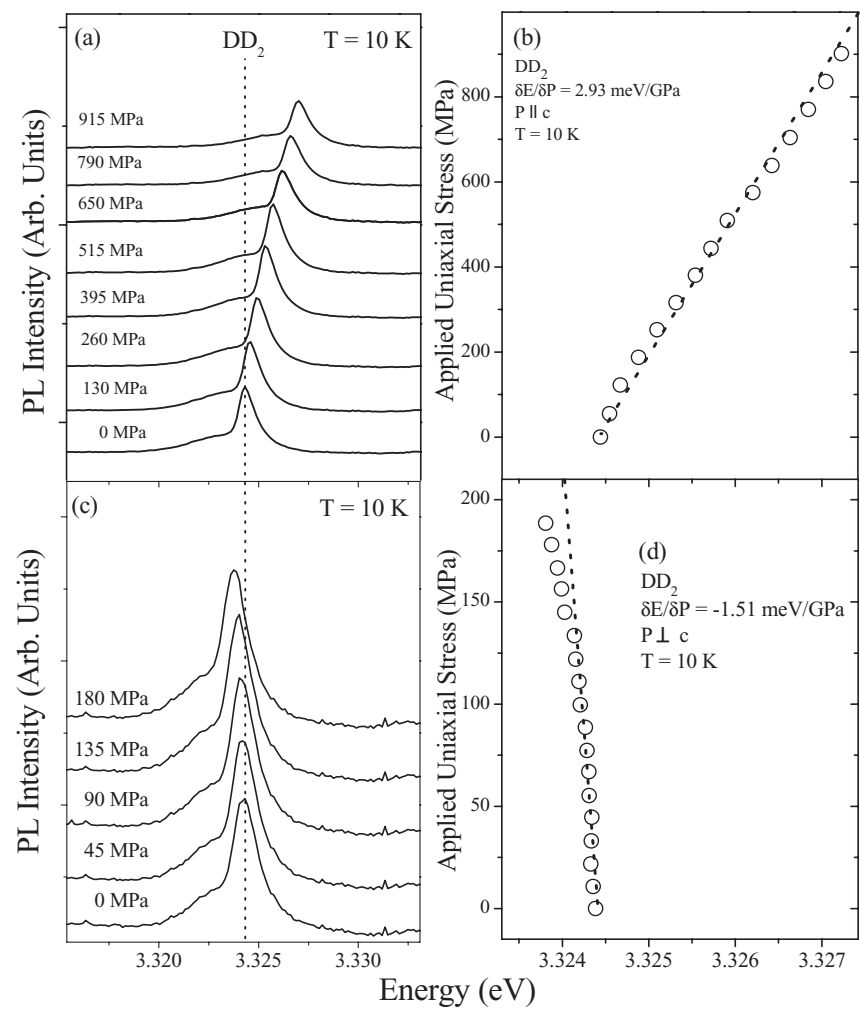

FIG. 2. (a) PL spectra and (b) energy of the line maxima of $\mathrm{DD}_{2}$ under uniaxial stress along the $c$ axis. The spectra and data for stress perpendicular to the $c$ axis applied along the [1-210] direction are shown in panels (c) and (d), respectively. Spectra have been vertically shifted for clarity. Dotted lines in panels (b) and (d) correspond to linear fits to the line energies. Note the different ranges for stress applied parallel and perpendicular to the $c$ axis. 


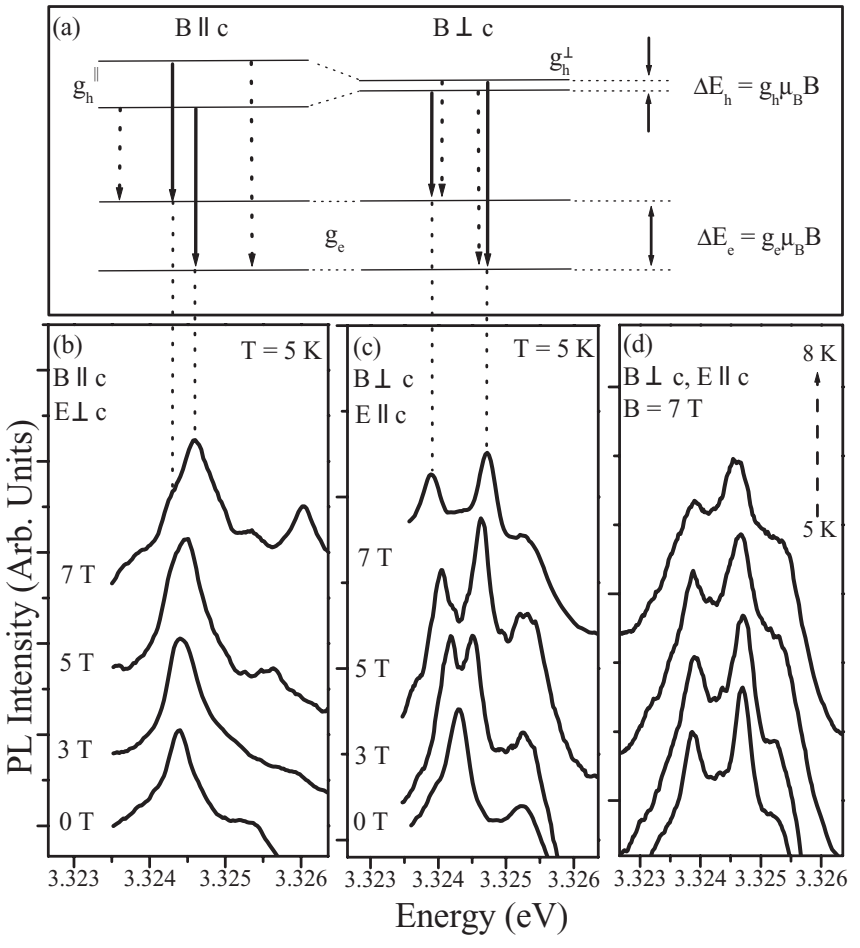

FIG. 3. (a) Schematic energy levels for the ground and excited states of a neutral DBX under magnetic fields parallel (left) and perpendicular (right) to the $\mathrm{ZnO} c$ axis. The solid vertical arrows indicate the allowed transitions, while dashed lines indicate forbidden transitions for the conditions used. The identifications of the observed lines with the allowed transitions are shown by the dotted lines. PL spectra are shown for the $\mathrm{DD}_{2}$ line measured in the magnetic field applied (b) parallel and (c) perpendicular to the $c$ axis for $\mathbf{B}=0,3,5$, and $7 \mathrm{~T}$. Spectra of the $\mathrm{DD}_{2}$ line for temperatures between 5 and $8 \mathrm{~K}$ and $\mathbf{B}=7 \mathrm{~T}$ are shown in panel (d) for $\mathbf{B} \perp \mathbf{c}$. The line just above $3.326 \mathrm{eV}$ in the 7-T spectrum of panel (b) is part of the $\mathrm{I}_{6}$ TES lines.

separation increases), and each component maintains the full width at half maximum of the zero field emission $(<0.3 \mathrm{meV})$. The absence of excited state thermalization is confirmed by the temperature-dependent Zeeman spectra shown in Fig. 3(d). Although only a limited temperature range was accessible, the spectra show an unchanging relative intensity between the split components as the temperature is changed. Hence, the splitting observed in Fig. 3(c) occurs mainly (if not exclusively) in the ground state of the transition. The significance of this finding is discussed later. The values obtained from the data for the splitting of $\mathrm{DD}_{2}$ under a magnetic field are $0.04 \mathrm{meV} / \mathrm{T}$ for $\mathbf{B} \| \mathbf{c}$ and $0.12 \mathrm{meV} / \mathrm{T}$ for $\mathbf{B} \perp \mathbf{c}$.

\section{DISCUSSION}

Radiotracer data presented in Ref. 3 led to the assignment of the $\mathrm{DD}_{2}$ feature as related to a single substitutional $\mathrm{Ge}_{\mathrm{Zn}}$ impurity. The very low thermal binding energy of $2.9 \mathrm{meV}$ for the center was reported in a later paper. ${ }^{6}$ The localization energy increases with temperature in a manner similar to that of $\mathrm{DD}_{1} / \mathrm{Y}_{0},{ }^{7}$ and the position of the line in the same spectral region as the group of $\mathrm{Y}$ lines with somewhat similar thermal properties, which differ from those of the well-understood I lines, suggests that these PL centers may constitute a distinct group with some as-yet-unknown common features distinct from those of the I lines. The data in Table I, however, do not all support this broad division of the PL into two groups of lines. For example, while the thermal binding energies of the DD/Y lines are quite similar to each other, and distinctly different from those of the I lines, anomalies in the intensity ratio of the LO phonon replicas to their corresponding zero phonon lines are also clear from the table. Similarly, the behavior of the lines under stress along the $c$ axis does not indicate two clearly defined and distinct groups. The shift rates for the I lines cluster $\sim 3 \mathrm{meV} / \mathrm{GPa}$ and those for the $\mathrm{Y}$ lines are $\sim 2$ $\mathrm{meV} / \mathrm{GPa}$, but $\mathrm{DD}_{2}$, as we point out later, behaves like the former. Accordingly, the question as to whether all DD/Y lines form a distinct group remains open, so in the discussion that follows, we compare and contrast the uniaxial stress and magneto-optical data we have obtained for the $\mathrm{DD}_{2}$ line to published results for DD/Y lines and for I lines.

\section{A. Uniaxial stress data analysis}

The main finding in the uniaxial stress measurements reported in Fig. 2 was that the $\mathrm{DD}_{2}$ line does not split under stress either parallel or perpendicular to the $c$ axis; therefore, there is neither electronic nor orientational degeneracy associated with the line. This general finding can be analyzed in detail by applying the generic results for the shift rates of transitions in wurtzite crystals under stress reported by McGlynn and Henry. ${ }^{13}$ From this, it is found that the absence of splitting means that the transitions involved must be between orbitally nondegenerate states at a defect of trigonal symmetry, similar to the case of substitutional donors that produce the I lines. Furthermore, the shift rate for stress parallel to the $c$ axis of $+2.93 \mathrm{meV} / \mathrm{GPa}$ lies in the range of values found for the I lines $(+2.79$ to $+3.37 \mathrm{meV} / \mathrm{GPa})$ and is significantly larger than the corresponding value for $\mathrm{DD}_{1} / \mathrm{Y}_{0}$ of $+2.09 \mathrm{meV} / \mathrm{GPa}^{7}$ Thus, although there are some clear parallels between the $\mathrm{DD}_{2}$ and the $\mathrm{DD}_{1} / \mathrm{Y}_{0}$ spectra, as pointed out earlier, they show quite different responses to stress along the $c$ axis. A shift rate similar to that for simple substitutional impurities, which produce the I lines, is consistent with the evidence from radiotracer work ${ }^{3,6,14}$ that the $\mathrm{Ge}$ atom occupies a $\mathrm{Zn}$ site and that the defect is a relatively simple "point" defect rather than a structural defect, as proposed for $\mathrm{DD}_{1} / \mathrm{Y}_{0}$.

The stress shift rates found in this paper for both stress directions, and in Ref. 7 for stress parallel to the $c$ axis, have the same signs but are of lower magnitudes than those published for bulk $\mathrm{ZnO}$ unresolved bound-exciton luminescence. ${ }^{12} \mathrm{~A}$ possible explanation is that these differences may result from crystal inhomogeneities that vary significantly from one $\mathrm{ZnO}$ crystal to another. ${ }^{15}$

\section{B. Zeeman spectroscopy data analysis}

The analysis of Zeeman data for bound excitons in II-VI crystals was first developed by Thomas and Hopfield ${ }^{16}$ for the case of CdS, and the same approach has been applied successfully to $\mathrm{ZnO} \cdot{ }^{17,18}$ In brief, the donor or acceptor nature of a bound-exciton center can be identified from the thermalization behavior of the line components under magnetic fields. ${ }^{18}$ For neutral DBXs, which consist of two 
electrons and a hole, the two electrons couple to form for a spin zero state and the hole is the only unpaired spin in the excited state. For the bare neutral donor, i.e., the ground state, there is an unpaired electron. Thus, the excited state splitting in DBXs is due to the hole (from the $\Gamma_{7} A$ valence band) and governed by the hole $g$ value $g_{h}$, while the ground state splitting is governed by the electron $g$ value $g_{e}$. The $g_{h}$ is highly anisotropic, with the value for fields perpendicular to the $c$ axis $g_{h}{ }^{\perp}$ being close to zero; ${ }^{19}$ therefore, the splitting for DBX features for $\mathbf{B} \perp \mathbf{c}$ is mostly due to $g_{e}$, i.e., to a ground state splitting. For fields parallel to the $c$ axis, the usual situation in $\mathrm{ZnO}$ is that $g_{h}<0$ and $\left|g_{h}\right|<g_{e}$, with the result that a DBX line splitting for $\mathbf{B} \perp \mathbf{c}$ is found to be larger than that observed for B || c. ${ }^{7,17}$ The schematic energy level diagram included in Fig. 3(a) incorporates all of these factors.

We now apply these rules to the case of $\mathrm{DD}_{2}$. The splitting for $\mathbf{B} \perp \mathbf{c}$ is larger than that for $\mathbf{B} \| \mathbf{c}$, and it occurs in the ground state, while for $\mathbf{B} \| \mathbf{c}$, we observe only a broadening of the line. Based on this characteristic behavior, we conclude that the $\mathrm{DD}_{2}$ line results from the recombination of a neutral DBX. This supports our earlier identification of the PL lines near $3.262 \mathrm{eV}$ as TES lines associated with $\mathrm{DD}_{2}$.

The values of both $g_{h}$ and $g_{e}$ are normally obtained by fitting angular dependence data to general equations that take account of anisotropy in both $g_{h}$ and $g_{e}$. Our data are limited to the special cases of $\mathbf{B} \| \mathbf{c}$ and $\mathbf{B} \perp \mathbf{c}$ only, but we can nevertheless estimate the values for $g_{h}$ if we assume that $g_{e}$ has a typical value and that the $g_{e}$ anisotropy is negligible, as has been found to be the case for $\mathrm{ZnO} .{ }^{17}$ The schematic energy level diagrams in Fig. 3(a) provide the necessary background for the estimation of $g$ values. The solid vertical arrows indicate the allowed transitions, while the dashed lines indicate forbidden transitions for the directions of the magnetic field $\mathbf{B}$ and the luminescence electric polarization vectors $\mathbf{E}$ used in the measurements. For $\mathbf{B} \| \mathbf{c}$, the separation of the observed transitions is governed by the value of $\left|g_{e}\right|$ - $\left|g_{h} \|\right|$, since the shifts in the ground and excited states are subtractive. In contrast, for $\mathbf{B} \perp \mathbf{c}$, the separation is determined by the value of $\left|g_{e}\right|+\left|g_{h}{ }^{\perp}\right|$, since the shifts in the ground and excited states in this case are additive. In addition, we can deduce the relative magnitudes of $\left|g_{e}\right|$ and $\left|g_{h} \|\right|$ from the relative intensity of the two lines in the B || c data. Since the lower energy line is the weaker one, it must originate on the upper level of the excited state, which has a lower population, and this requires $\left|g_{h}{ }^{\|}\right|<\left|g_{e}\right|$. Regarding the sign of $g_{h} \|$, we do not have polarization data on which to base a decision, and in the calculation that follows we assume that it is negative, as is the case for $\mathrm{ZnO} \mathrm{DBXs}$ studied to date. . $^{71}$

From the data presented in Fig. 3(b) and 3(c), we found that the splitting of $\mathrm{DD}_{2}$ under a magnetic field is $0.04 \mathrm{meV} / \mathrm{T}$ for B $\|$ c and $0.12 \mathrm{meV} / \mathrm{T}$ for $\mathbf{B} \perp$ c. Starting with the case of $\mathbf{B} \perp \mathbf{c}$, our data give the effective $g$ value for the exciton $g_{\text {exc }}=$ 2.07. For $\mathbf{B} \| \mathbf{c}$, the value obtained is $g_{\text {exc }}=0.69$. If we assume that $g_{e}=2.02$ (the value reported for $\mathrm{DD}_{1} / \mathrm{Y}_{0}$ ), then we obtain $g_{h}{ }^{\|}=-1.33$ and $g_{h}{ }^{\perp}=0.05$. The corresponding values for the case of $\mathrm{DD}_{1} / \mathrm{Y}_{0}$ in Ref. 7 are $g_{h}{ }^{\|}=-1.30$ and $g_{h}{ }^{\perp}=$ 0.15 . We can make the same calculation assuming a $g_{e}$ value of 1.90, which is in the range found for the I lines, and from this we obtain $g_{h} \|=-1.21$ and $g_{h}{ }^{\perp}=0.17$. These compare to $g_{h} \|=-1.21$ and $g_{h}{ }^{\perp}=0.1$ for $\mathrm{I}_{4}$ and $g_{h} \|=-1.27$ and $g_{h}{ }^{\perp}=0.06$ for $\mathrm{I}_{9} \cdot{ }^{17}$ Overall, the $g$-value estimates, unlike the stress shift rates, do not clearly discriminate between the I line and the $\mathrm{DD} / \mathrm{Y}$ line nature for $\mathrm{DD}_{2}$. However, this is not unexpected, as the $g$ values are dominated by electron and hole spin. Regardless of the detailed electron and hole $g$ values, the main finding of the magneto-optical studies is that the $\mathrm{DD}_{2}$ line shows behavior characteristic of neutral DBX emission.

\section{A model for the $\mathrm{DD}_{2}$ recombination center}

We now consider several models for the $\mathrm{DD}_{2}$ center that possess the trigonal symmetry and neutral DBX character indicated by the uniaxial stress and magneto-optical data.

\section{Recombination at neutral $\mathrm{Ge}_{\mathrm{Zn}}$ impurities}

The simplest case to consider is that the center consists of isolated $\mathrm{Ge}_{\mathrm{Zn}}$ atoms. This satisfies the symmetry requirement and is consistent with the radioactivity data published previously ${ }^{3,6}$ and, in general terms, with $\mathrm{D}^{0} \mathrm{X}$ recombination, since $\mathrm{Ge}_{\mathrm{Zn}}$ would act as a donor. However, as a group IV impurity on a group II $\mathrm{Zn}$ site, Ge acts as a double donor, so the details of the exciton states differ from those of a single donor and they are not consistent with the magneto-optical data. Furthermore, it is difficult to reconcile a large exciton localization energy of $53 \mathrm{meV}$ with a center containing three electrons and a hole.

The possibility that the luminescence is due to the recombination of a weakly localized hole with an electron on a neutral Ge double donor exists as well, since such an entity consists of two electrons and a hole, equivalent to a DBX. This entity could also be viewed as an exciton bound to a singly ionized double donor, so it is consistent with the magneto-optical data. These alternatives were considered as possible models for the $\mathrm{DD}_{1} / \mathrm{Y}_{0}$ defect by Wagner et al., ${ }^{7}$ who provided a detailed analysis of the thermal and spectral characteristics that follow. If we consider this possibility for the case of $\mathrm{DD}_{2}$, the main difficulty lies with the positions of the TES lines. As the ground state after recombination is a singly ionized double donor, TES lines associated with $\mathrm{DD}_{2}$ are expected to lie at a large separation from the principal line. The only lines we have observed as possible TES lines are $\sim 63 \mathrm{meV}$ below $\mathrm{DD}_{2}$. If we assign this energy to the $1 s \rightarrow 2 p$ transition of an electron bound to a $\mathrm{Ge}^{2+}$ core, then we obtain an estimated binding energy for the second electron on the Ge double donor of $\sim 80 \mathrm{meV}$. If we apply a simple heliumlike model to the double donor, with the neutral state being $\mathrm{He}^{0}$ and the singly ionized state being $\mathrm{He}^{+}$, then the (first) electron on $\mathrm{He}^{0}$ has a binding energy that is only $1 / 4$ of that of the (second) electron on $\mathrm{He}^{+}$. In the present case, this implies that the first electron on the Ge double donor is bound by only $\sim 20 \mathrm{meV}$, which is extremely shallow and less than half of the typical effective mass donor-binding energies deduced for the common single donor impurities. ${ }^{7}$ Still, it remains a possibility. Bearing in mind the very weak $\mathrm{DD}_{2}$ thermal binding energy of only $2.9 \mathrm{meV},{ }^{6}$ and its shallow donorlike stress behavior, we cannot exclude the recombination of a hole with an electron on the neutral $\mathrm{Ge}$ double donor as the origin of the $\mathrm{DD}_{2}$ luminescence. 


\section{A compound Ge defect in $\mathrm{ZnO}$}

We now consider the possibility that $\mathrm{DD}_{2}$ results from a combination of a $\mathrm{Ge}_{\mathrm{Zn}}$ substitutional impurity and another simple impurity or defect, i.e., from a compound defect in which the Ge impurity is just one of the components. Examples of such a defect include $\mathrm{Ge}_{\mathrm{Zn}}$ in combination with a nearby vacancy or acceptor impurity such as $\mathrm{Cu}_{\mathrm{Zn}}$ or $\mathrm{Li}_{\mathrm{Zn}}$. These latter cases are both deep acceptors in $\mathrm{ZnO},{ }^{20,21}$ which could create local potentials to attract diffusing $\mathrm{Ge}$ impurities into their vicinity in the crystal. In considering these various possibilities, the trigonal symmetry and donor nature established from experiment must be paramount. We first consider Ge-acceptor pairs, where the single acceptor (e.g., suggested earlier as $\mathrm{Cu}$ or $\mathrm{Li}$ ) partially compensates the $\mathrm{Ge}_{\mathrm{Zn}}$ double donor. In order to retain trigonal symmetry, the $\mathrm{Cu}$ or $\mathrm{Li}$ atom would have to lie along the $c$ axis in a $\mathrm{Zn}$ site, and the closest such site lies one lattice constant from the location of the Ge atom. In a similar fashion, instead of an acceptor impurity, there could exist a $\mathrm{Zn}$ vacancy $\mathrm{V}_{\mathrm{Zn}}$ along the $c$ axis. The electrical behavior of $\mathrm{V}_{\mathrm{Zn}}$ is that of an acceptor, ${ }^{22,23}$ so here too the required trigonal symmetry and donor character for $\mathrm{DD}_{2}$ could be produced by $\mathrm{V}_{\mathrm{Zn}}$ on the $c$ axis close to the $\mathrm{Ge}_{\mathrm{Zn}}$ impurity. However, the $\mathrm{O}$ vacancy $\left(\mathrm{V}_{\mathrm{O}}\right)$ acts as a donor, so it does not provide the compensation required for $\mathrm{Ge}_{\mathrm{Zn}} \cdot{ }^{24} \mathrm{In}$ this model, the recombining electron and hole wavefunctions have different distributions over the defect, and the electron and hole could be expected to have different individual binding energies to the defect, with the more loosely bound particle mainly determining the thermal binding energy and the other mainly determining the spectral position.

The involvement of $\mathrm{Cu}$ or $\mathrm{Li}$ could, in principle, be examined by implanting $\mathrm{Ge}$ into starting $\mathrm{ZnO}$ material with differing amounts of these impurities. However, commercially available $\mathrm{ZnO}$ tends to have significant levels of these impurities already present, making the use of widely varying $\mathrm{Cu}$ or $\mathrm{Li}$ concentrations difficult to achieve. Annealing in a $\mathrm{Zn}$-rich atmosphere favors the destruction of $\mathrm{Zn}$ vacancies, and this might provide evidence to support the involvement of $\mathrm{Zn}$ vacancies if the $\mathrm{DD}_{2}$ signal was found to decrease significantly under such conditions.

\section{Recombination at structural defects}

Given its similarities in some respects to the $\mathrm{DD}_{1} / \mathrm{Y}_{0}$ center, we consider the possibility that $\mathrm{DD}_{2}$ involves $\mathrm{D}^{0} \mathrm{X}$ recombination in the vicinity of a structural defect, as has been suggested for $\mathrm{DD}_{1} / \mathrm{Y}_{0}$ by Wagner et al. ${ }^{7}$ These authors provided a detailed qualitative model based on a defect core consisting of two adjacent donor centers producing the equivalent of a single DBX following the capture of an exciton or a hole (depending on the initial charge state of the core) under illumination. One of the main arguments presented in favor of such an extended defect model is the significantly different uniaxial pressure coefficient of $\mathrm{DD}_{1} / \mathrm{Y}_{0}$ in comparison to that of the I lines for pressure parallel to the $c$ axis. This finding, according to those authors, "indicates a distinctly different structure of the $\left[\mathrm{DD}_{1} / \mathrm{Y}_{0}\right.$ defect cores in comparison to the shallow bound excitons." If we apply this reasoning (in reverse) to the case of $\mathrm{DD}_{2}$, then the similar uniaxial pressure coefficients we found for $\mathrm{DD}_{2}$ and the I lines indicate similar defect cores for $\mathrm{DD}_{2}$ and the I lines; i.e., the pressure coefficient data favor the identification of $\mathrm{DD}_{2}$ with a simple substitutional impurity.

In principle, it is possible that extended crystal defects could result in partial compensation of the $\mathrm{Ge}_{\mathrm{Zn}}$ double donors, thereby creating a single donor center, and the strain effects of a structural defect could contribute to the electron and hole becoming bound in the complex without destroying the trigonal symmetry of the core of the exciton complex. On balance, however, we believe that this model is unnecessarily complicated for $\mathrm{DD}_{2}$ and that the defect that binds the exciton is more likely to involve a single Ge impurity atom in a suitable charge state, possibly in conjunction with another impurity or a vacancy.

\section{SUMMARY}

The properties of the Ge-related $\mathrm{DD}_{2}$ PL line at $3.324 \mathrm{eV}$ in $\mathrm{ZnO}$ have been examined using uniaxial stress and Zeeman measurements. The uniaxial stress data show that the line is due to transitions between orbitally nondegenerate states at a defect of trigonal symmetry, and the Zeeman data show that the line has the characteristics typical of DBX recombination. The line is found to have some parallels to the $\mathrm{Y}$ lines associated with structural crystal defects, yet it shows some similarities to the well-understood bound-exciton I lines that arise from simple substitutional impurities. In principle, the defect responsible for $\mathrm{DD}_{2}$ could be as simple as a $\mathrm{Ge}_{\mathrm{Zn}}$ double-donor impurity that captures a hole with a very low binding energy. Alternatively, a neutral donor produced by a compound defect involving $\mathrm{Ge}_{\mathrm{Zn}}$ and some other crystal imperfection, such as a nearby acceptor impurity, Zn-site vacancy, or structural defect that does not destroy the trigonal symmetry, would also fit the experimental data. Distinguishing between these possibilities, and explaining the anomalous properties of loosely bound but spectrally deep bound-exciton luminescence lines, is one of the major challenges at this time in $\mathrm{ZnO} \mathrm{PL}$ spectroscopy.

\section{ACKNOWLEDGMENTS}

This paper has been supported by Science Foundation Ireland under Project No. 08/RFP/PHY1558 and by the European Community as an integrating activity under both Support of Public and Industrial Research Using Ion Beam Technology (Project No. 227012) and European Nuclear Science and Applications Research (Project No. 262010). Thanks also to Markus R. Wagner of the Technische Universität Berlin for helpful discussion.

\footnotetext{
*Corresponding author: martin.henry@dcu.ie

${ }^{1}$ C. Klingshirn, J. Fallert, H. Zhou, J. Sartor, C. Thiele, F. Maier-

Flaig, D. Schneider, and H. Kalt, Phys. Status Solidi B 247, 1424

(2010).
}

${ }^{2}$ B. K. Meyer, H. Alves, D. M. Hofmann, W. Kriegseis, D. Forster, F. Bertram, J. Christen, A. Hoffmann, M. Strassburg, M. Dworzak, U. Haboeck, and A. V. Rodina, Phys. Status Solidi B 241, 231 (2004). 
${ }^{3}$ K. Johnston, M. O. Henry, D. McCabe, E. McGlynn, M. Dietrich, E. Alves, and M. Xia, Phys. Rev. B 73, 165212 (2006).

${ }^{4}$ S. Müller, D. Stichtentoth, M. Urhmacher, H. Hofsäss, C. Ronning, and J. Röder, Appl. Phys. Lett. 90, 012107 (2007).

${ }^{5}$ D. C. Reynolds and T. C. Collins, Phys. Rev. 185, 1099 (1969).

${ }^{6}$ K. Johnston, J. Cullen, M. O. Henry, E. McGlynn, and M. Stachura, Phys. Rev. B 83, 125205 (2011)

${ }^{7}$ M. R. Wagner, G. Callsen, J. S. Reparaz, J.-H. Schulze, R. Kirste, M. Cobet, I. A. Ostapenko, S. Rodt, C. Nenstiel, M. Kaiser, A. Hoffmann, A. V. Rodina, M. R. Phillips, S. Lautenschläger, S. Eisermann, and B. K. Meyer, Phys. Rev. B 84, 035313 (2011).

${ }^{8}$ A. Schildknecht, R. Sauer, and K. Thonke, Phys. B 205, 340 (2003). ${ }^{9}$ J. L. Lyons, A. Janotti, and C. G. Van de Walle, Phys. Rev. B 80, 205113 (2009).

${ }^{10}$ C. O’Morain, K. G. McGuigan, M. O. Henry, and J. D. Campion, Meas. Sci. Technol. 3, 337 (1992).

${ }^{11}$ O. Akimoto and H. Hasegawa, Phys. Rev. Lett. 20, 916 (1968).

${ }^{12}$ D. W. Langer, R. N. Euwema, K. Era, and T. Koda, Phys. Rev. B 2 , 4005 (1970)

${ }^{13}$ E. McGlynn and M. O. Henry, Phys. Rev. B 76, 184109 (2007).
${ }^{14}$ U. Wahl, E. Rita, J. G. Correia, A. C. Marques, E. Alves, and J. C. Soares, Phys. Rev. Lett. 95, 215503 (2005).

${ }^{15} \mathrm{M}$. R. Wagner (private communication, 2012).

${ }^{16}$ D. G. Thomas and J. J. Hopfield, Phys. Rev. 128, 2135 (1962).

${ }^{17}$ A. V. Rodina, M. Strassburg, M. Dworzak, U. Haboeck, A. Hoffmann, A. Zeuner, H. R. Alves, D. M. Hofmann, and B. K. Meyer, Phys. Rev. B 69, 125206 (2004).

${ }^{18}$ G. Blattner, C. Klingshirn, R. Helbig, and R. Meinl, Phys. Status Solidi B 107, 105 (1981).

${ }^{19}$ M. R. Wagner, J.-H. Schulze, R. Kirste, M. Cobet, A. Hoffmann, C. Rauch, A. V. Rodina, B. K. Meyer, U. Röder, and K. Thonke, Phys. Rev. B 80, 205203 (2009).

${ }^{20}$ J. J. Lander, J. Phys. Chem. Solid. 15, 324 (1960).

${ }^{21}$ H. Kamimura and A. Yariv, Bull. Am. Phys. Soc. 8, 23 (1963).

${ }^{22}$ C. R. A. Catlow, S. A. French, A. A. Sokol, A. A. Al-Sunaidi, and S. M. Woodley, J. Comput. Chem. 29, 2234 (2008).

${ }^{23}$ X. J. Wang, L. S. Vlasenko, S. J. Pearton, W. M. Chen, and I. A. Buyanova, J. Phys. D Appl. Phys. 42, 175411 (2009).

${ }^{24}$ A. Janotti and C. G. van de Walle, Appl. Phys. Lett. 87, 122102 (2005). 